\title{
Blended Learning: Auf dem langen Weg zur neuen Qualität in der Lehre
}

\author{
Maika Büschenfeldt, Margit Scholl
}

Zusammenfassung

Blended Learning ist ein integriertes Lernkonzept, das die Möglichkeiten des Online Lernens in Kombination mit den traditionellen Formen des Präsenzlernens in optimaler Weise verbinden soll. Blended Learning ist herausfordernd, weil die konsequente Umsetzung auch Innovationen in der Lehre einschließt und zur Veränderung der Lehrkultur und Lernformen führt. In Weiterentwicklung des Forschungsansatzes des EU-Projektes COMBLE identifizieren wir drei relevante Bereiche zur Verankerung von Blended Learning: die institutionelle und organisatorische Einbindung, die technische Infrastruktur mit ihren technischen Support und die didaktische Infrastruktur mit didaktischen Unterstützungsmaßnahmen.

\section{Abstract}

Blended Learning is an integrated learning concept that combines the possibilities of online learning with traditional forms of classroom learning in an optimal way. Blended learning is challenging because the consistent implementation of this learning concept also includes innovations in teaching, leading to a change in teaching culture and ways of learning. In developing the research approach of the EU project COMBLE further, we identify three relevant areas for the anchoring of Blended Learning: the institutional and organizational integration, the technical infrastructure with technical support and the educational infrastructure with educational assistance.

\section{Einleitung}

Lebenslanges Lernen zählt seit Jahren zu den gesellschaftspolitischen Herausforderungen und zu den vorrangigen bildungspolitischen Aufgaben. Im Rahmen der »Qualifizierungsinitiative für Deutschland « [1] gehört die Durchlässigkeit zwischen beruflicher Ausbildung und Hochschulbildung zu den zentralen Zielsetzungen der Bildungspolitik. Die Hochschulen sind damit aufgefordert, nicht nur den Hochschulzugang und die Anrechenbarkeit beruflicher Qualifikationen zu erleichtern, sondern auch für beruflich Qualifizierte berufsbegleitende Studienangebote als Beitrag zum Lebenslangen Lernen zu schaffen. (BMBF 2008)

Die Erwartung an die Hochschulen, ihren Beitrag zum Lebenslangen Lernen und zur Durchlässigkeit zu leisten, liegt durchaus im Eigeninteresse, da der erwartete Rückgang der Studierendenzahlen im Zuge des Demografischen Wandels dazu zwingt, nach neuen Zielgruppen Ausschau zu halten. Für die Hochschulen bedeutet dies zunächst zweierlei:

- Sie haben sich auf neue Zielgruppen und auf die Rolle als Anbieter beruflicher bzw. berufsbegleitender Weiterbildungsangebote einzustellen. Daraus erwachsen neue Anforderungen an Professionalität, Zertifizierung und Praxisorientierung in der Gestaltung und Durchführung von Weiterbildungsangeboten und Fortbildungslehrgängen. Zu den Anforderungen gehört zusätzlich die Umstellung auf flexiblere Lehrangebote, die gleichsam das Methodenrepertoire und die Organisation der bislang praktizierten Präsenzlehre zur Disposition stellen. Ebenso werden im Zuge einer stärkeren Internationalisierung zukünftig verstärkt kulturelle Aspekte zu berücksichtigen sein.

- Neue Anforderungen ergeben sich aber auch aus der Entwicklung neuer Technologien und der Erschließung neuer Kommunikationswege, die unter dem Stichwort Web 2.0 oder Social Media nicht ganz zu Unrecht die Erwartung wecken, durch deren Nutzung eine neue Qualität in der Aus-, Fort- und Weiterbildung zu erreichen. Da unsere Gesellschaft sich durch das Internet und seine Dienste als neue Kommunikationsform verändert, stellt sich somit die Frage, ob sich diese Entwicklung bislang angemessen in der Hochschullehre niedergeschlagen hat. Darüber hinaus führt diese technisch orientierte Modernisierung unserer Gesellschaft zu erhöhten Anforderun- 
gen an eine umfassende Informationssicherheit und es wird in den Bildungsstätten notwendig, sich mit diesen Fragen kritisch auseinanderzusetzen. Dabei gilt es zu prüfen, inwieweit sich die tradierten Ansätze und Regeln der realen, visuellen Welt in eine zunehmend virtuelle Welt übertragen lassen oder ob neue Ansätze geschaffen werden müssen.

Mit dem Anspruch, flexiblere Lernangebote unter Ausnutzung moderner Kommunikationstechnologien zu entwickeln, kommen daher vielfältige Anforderungen auf die Hochschulen zu. E-Learning wird für berufsbegleitende Angebote wie das Fernstudium zum »muss « und für die klassische Präsenzlehre zur ernsthaften Option, weshalb sich die Hochschulen diesen Anforderungen und Prozessen stellen sollten.

E-Learning galt noch vor einigen Jahren als Bildungsform des 21. Jahrhunderts, von der man in der allgemeinen Hypestimmung annahm, dass diese die traditionellen Bildungsformen vollständig verdrängen werde. Inzwischen ist die anfängliche Euphorie der Erkenntnis gewichen, dass E-Learning die traditionellen Bildungsformen nicht ersetzen, aber sinnvoll unterstützen und zu deren Weiterentwicklung beitragen kann. E-Learning wird in diesem Sinne zum Blended Learning (BL), d. h. zur sinnvollen Kombination von Präsenz- und Online-Lehre. Die Umsetzung von Blended Learning ist keine triviale Aufgabe, sondern mit erheblichen Anstrengungen verbunden, da ein solches Vorhaben weitaus mehr erfordert als die Bereitstellung der technischen Infrastruktur durch den Aufbau einer E-Learning Plattform.

Wie ein solches Ziel erreicht werden kann, ist Gegenstand dieses Artikels. Ausgangspunkt bilden die aktuellen Anforderungen an die Hochschullehre (Abschnitt 2) und die Frage, was Gegenstand einer Blended Learning Strategie als Grundlage für den Aufbau einer didaktischen Infrastruktur sein kann (Abschnitt 3). Der Aufbau, die Bedeutung und Nutzung der technischen Infrastruktur beschreibt Abschnitt 4 am Beispiel der Projekte Virtual Campus und learn@wildau. In diesem Abschnitt wird die Einsatzbereitschaft für Blended Learning an der TH Wildau anhand der Auswertung von Nutzungsdaten der zentralen E-Learning Plattform untersucht. Konkrete Umsetzungsschritte und didaktische Überlegungen zur Etablierung von Blended Learning skizziert Abschnitt 5. Fazit und Ausblick folgen im letzten Abschnitt.

\section{Die Ebenen des Blended Learning}

Im EU-Projekt COMBLE [2] wurde bereits ein formales Modell als Instrument zur Prüfung der »Blended Learning Readiness « entwickelt. In diesem Modell werden über die Unterscheidung der Hauptakteursgruppen die verschiedenen Ebenen des Blended Learning identifiziert und die Frage in den Mittelpunkt gestellt, inwieweit eine Institution überhaupt die notwendigen Voraussetzungen für das Blended Learning erfüllt.

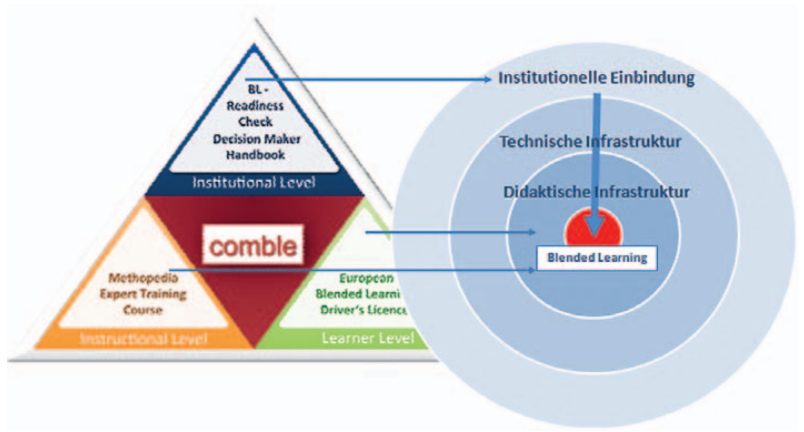

Abb. 1: Übertragung der Erkenntnisse aus COMBLE auf eine konkrete Einführungsstrategie für Blended Learning

In Weiterentwicklung des COMBLE Ansatzes möchten wir in diesem Artikel drei große Bereiche hervorheben, die wir als Voraussetzung für die Etablierung von Blended Learning in einer Organisation wie der $\mathrm{TH}$ Wildau ansehen:

\section{(a) Institutionelle Einbindung}

Eine Blended Learning Strategie kann erfolgreich nur entwickelt und umgesetzt werden, wenn Blended Learning institutionell eingebunden wird (»Institutional Level « in COMBLE). Konkret bedeutet dies, dass die dazu nötigen Ressourcen (auch auf Ministerialebene) bereitgestellt und Grundsatzfragen wie Mitwirkungsund Akzeptanzförderung einbezogen werden. Hier sind insbesondere die Entscheidungsträger gefragt, die den Willen zur Neugestaltung mittragen und die Erkenntnis teilen, dass die Einführung von Blended Learning über die erfolgreiche Implementierung einer Lernplattform hinaus, weitreichende Veränderungen in der Organisation und der Lernkultur erfordert.

\section{(b) Technische Infrastruktur}

Als Beitrag der technischen Infrastruktur spielen E-Learning Plattformen eine zentrale Rolle. Die Existenz einer zentralen, hochschulweiten Lernplattform hat sich inzwischen vom Status des exotischen Beiwerks zum festen Angebot vieler Hochschulen entwickelt und gilt als 
wichtiger Standortfaktor in der Konkurrenz der Hochschulen untereinander. Herausforderung ist hier nicht nur die technisch einwandfreie Implementierung einer Plattform, sondern auch die Aufgabe, die nötige Akzeptanz unter Studierenden und Lehrenden herzustellen. Dies setzt eine aktive Hilfe bei der Integration der Lerntechnologie in die laufenden Lehrveranstaltungen voraus. Aktive Hilfe kann nur geleistet werden, wenn die Nutzung der Plattform dauerhaft durch einen gut organisierten und personell ausreichend ausgestatteten Support unterstützt wird und auch Mittel zur technischen Weiterentwicklung der Plattform bereitstehen.

\section{(c) Didaktische Infrastruktur}

Die Bereitstellung einer E-Learning Plattform als technisches Service Angebot ist eine wichtige Voraussetzung, markiert jedoch nicht das Ende, sondern erst den Anfang der Entwicklung, die auf eine neue Qualität und Formvielfalt der traditionellen Lehre hinausläuft. Wir stehen damit vor einem Transformationsprozess, der von der traditionellen Präsenzlehre in die onlinegestützte Lehre führt und beides miteinander verbindet. Dieser Wandel sollte nicht planlos verlaufen, sondern von einer gezielten Blended Learning Strategie (BL-Strategie) flankiert werden. Diese Strategie berührt im COMBLE Dreieck den »Instructional Level« mit der Zielgruppe der Lehrenden und den »Learners Level« mit der Zielgruppe der Studierenden (siehe Abb.1). Gegenstand dieser Strategie ist die Organisation der didaktischen Beratung, Weiterbildung und Unterstützung. Dies beinhaltet auch die konkrete Gestaltung des Transformationsprozesses von der Präsenzveranstaltung zum Blended Learning, den Erwerb und die Sicherung von Methodenkenntnissen sowie die Evaluation und Qualitätssicherung der Lehrangebote. Die BL-Strategie erschließt den Weg in den Aufbau einer didaktischen Infrastruktur, die für Lehrende und Lernende den Rahmen und die notwendige Unterstützung in der Neugestaltung der Lehre bietet.

\section{Gegenstand einer Blended Learning Strategie}

\subsection{E-Learning und Blended Learning}

E-Learning steht als Oberbegriff für eine Vielzahl unterschiedlicher Lernformen und Technologien, die nur gemeinsam haben, dass Lehr- und Lernprozesse durch elektronische Medien unterstützt werden. (Kep- ser 2010: 200) Unter eine solch allgemeine Definition fällt somit nicht nur das Online gestützte Lernen, sondern auch die frühen Ansätze des Offline-Lernens am Computer z. B. mit Lernprogrammen auf DVD. Eine präzisere, allgemein anerkannte Definition des Begriffs steht noch aus. Das mag seinen Grund in der Vielgestaltigkeit dieses Feldes haben, die auch die Suche nach allgemeinen Aussagen über das E-Learning mehr oder weniger unmöglich macht. Schulmeister bemerkt dazu (Schulmeister 2005: 477):

»Es können keine allgemeinen Aussagen über E-Learning gemacht werden, da E-Learning-Angebote sich gravierend in Zielen, Szenarien, Lernumgebungen, Methoden und Lernobjekten unterscheiden «

Der Begriff Blended Learning bezieht sich auf den organisatorischen Aspekt des E-Learnings, thematisiert das Verhältnis von Präsenz- und Onlinelehre sowie die gezielte Aktivierung und Beteiligung der Lernenden. In der Definition von (Sauter u. a. 2004) wird Blended Learning als integriertes Lernkonzept in Verbindung mit den Technologien des Internets beschrieben:

"Blended Learning ist ein integriertes Lernkonzept, das die heute verfügbaren Möglichkeiten der Vernetzung über Internet oder Intranet in Verbindung mit »klassischen « Lernmethoden und-medien in einem sinnvollen Lernarrangement optimal nutzt. Es ermöglicht Lernen, Kommunizieren, Informieren und Wissensmanagement, losgelöst von Ort und Zeit in Kombination mit Erfahrungsaustausch, Rollenspiel und persönlichen Begegnungen im klassischen Präsenztraining. «(Sauter et al. 2004: 68)

In dieser Definition finden sich bereits bedeutsame Hinweise auf das Potenzial und die Besonderheit des Blended Learning Ansatzes.

\subsection{Die Potenziale des Internets}

Das Internet mit seinen Diensten wird zum Medium des Blended Learnings und eröffnet der Lehre die Möglichkeit, den räumlichen und zeitlichen Beschränkungen zumindest teilweise zu entfliehen. Das Potenzial dieser Verbindung liegt somit in der Flexibilisierung durch die Entkopplung des Lernens von Zeit und Raum. In der Literatur wird das didaktische Potenzial des Internets darüber hinaus entlang der Funktionen im Lehr- und Lernprozess beschrieben: (Hof 2009: 73)

- Learning by Distributing, gründet im Potenzial beliebiger Vervielfältigung und Verteilung digitaler Formate. Der Lernprozess wird durch das Bereitstellen und/oder den Austausch von Informationen und 
Materialien unterstützt. Dies stellt für uns lediglich die Grundlage des Blended Learning dar.

- Learning by Interacting, gründet im Potenzial dynamischer Webanwendungen oder Digitalmedien mit den Lernenden zu interagieren. Der Lernprozess kann auch ohne direkte Präsenz eines Lehrenden interaktiver gestaltet werden, findet jedoch in den beschränkten Reaktionsmöglichkeiten der eingesetzten Software seine Grenzen. Dies ist ein notwendiger weiterer Schritt, den Lehrende und Lernende gehen sollten und setzt bereits ein verändertes Methodenund Handlungskonzept voraus.

- Learning by Collaboration, gründet im Potenzial des Internets als Kommunikationsmedium wirksam zu werden und bezeichnet Lernansätze, die auf der netzwerkartigen Interaktion von Personen untereinander basieren. Mit dieser Entwicklungsstufe werden die Rollen der Lehrenden und Lernenden verändert und an neuere didaktische Methoden und Lernformen angeknüpft.

\subsection{Blended Learning als integriertes Lernkonzept} Blended Learning ist ein integriertes Lernkonzept, das die Möglichkeiten des Online-Lernens in Kombination mit den traditionellen Formen des Präsenzlernens in optimaler Weise verbinden soll. Dies beschreibt zwar wichtige Aspekte der Anforderungen, lässt aber offen, wie sich die Potenziale des Internets und die klassischen Formen der Präsenzlehre als integratives Gesamtkonzept kombinieren lassen. Gegenstand einer BL-Strategie muss daher sein, eine didaktische Infrastruktur aufzubauen, die es Lehrenden ermöglicht, das OnlineLernen und die Präsenzlehre als integriertes Konzept zu einer neuen Qualität der Lehre zu verbinden. Die auf die reine Präsenzlehre zugeschnittenen Lernformen wie Vorlesung, Seminar oder Übung stehen damit in ihrer klassischen Ausprägung zur Disposition und es stellt sich die Frage, inwieweit diese durch neue Lernformen ergänzt und selbst modifiziert werden sollten. Dieser Weg geht mit Veränderungen in den Abläufen und Raumnutzungen einher, so dass ein didaktisches Konzept ohne organisatorische Einbindung sehr schnell an seine Grenzen stoßen kann. In Anlehnung an das skizzierte dreistufige Modell in Abb. 1 soll im Folgenden die Einsatzbereitschaft für Blended Learning an der TH Wildau untersucht werden. Ausgehend von der Auswertung der Nutzungsdaten der E-Learning Plattform soll dabei insbesondere der Aufbau einer didaktischen Infrastruktur in den Mittelpunkt gestellt werden.

\section{E-Learning an der TH-Wildau: die technische Infrastruktur als Basis}

Die konkrete Einführung und Einrichtung einer hochschulweiten E-Learning Plattform an der TH Wildau begann mit dem Infrastruktur-Projekt Virtual Campus und erfolgte danach im Zeitraum von Juni 2009 bis Juni 2010 in enger Zusammenarbeit mit dem Hochschulrechenzentrum unter dem Programmnamen learn@ wildau. Ziel war es, die vielen Insellösungen der Hochschule durch eine einheitliche und supportgestützte ELearning-Infrastruktur abzulösen, um damit eine technische Basis für die Online-Unterstützung der Lehre aller Fachbereiche im Direkt- und Fernstudium zu schaffen.

\subsection{Die Lernplattform}

Als Learning-Management-System (LMS) wird die Open Source Software Moodle verwendet, die von einer internationalen Community entwickelt und sowohl an Schulen als auch an Universitäten weltweit genutzt wird. Kernstück dieses LMS sind Kursräume, die für die Lehrenden mehr als 20 konfigurierbare Aktivitäten bereithalten, um den Lernprozess zu unterstützen. Dazu zählen u. a. eine umfangreiche Ressourcenverwaltung, Foren, Glossare, Wikis, Aufgaben, Tests, Datenbanken oder Blogs.

Seit dem Wintersemester 2009/10 läuft die Plattform im Normalbetrieb, wird vom Hochschulrechenzentrum der TH Wildau gehostet und durch eine wissenschaftliche Projektstelle mit studentischer Unterstützung als Support betreut.

\subsection{Nutzung der Plattform}

Eine Einschätzung der Nutzung und Funktionsweise der Moodle Plattform soll im Folgenden auf der Grundlage der anonymen Auswertung der Logdaten, Datenbankinhalten und Supportanfragen nachvollzogen werden. Grob unterscheiden lassen sich zunächst Aussagen über die Häufigkeit der Zugriffe auf die Lernmodule der Plattform (Aktivitäten), die einen Eindruck über die Intensität der Nutzung vermitteln. Um das Bild abzurunden, soll anhand der Zugriffsverteilung auf die verschiedenen Module ebenfalls nachvollziehbar gemacht werden, inwieweit bislang auch interaktive und kollaborative Funktionen der Plattform genutzt wurden.

Im Zeitraum vom Projektbeginn im Juni 2008 bis Ende des Sommersemesters 2010 wurden auf der ELearning Plattform der TH Wildau insgesamt 1,25 Mio. Aktivitäten [3] geloggt. 


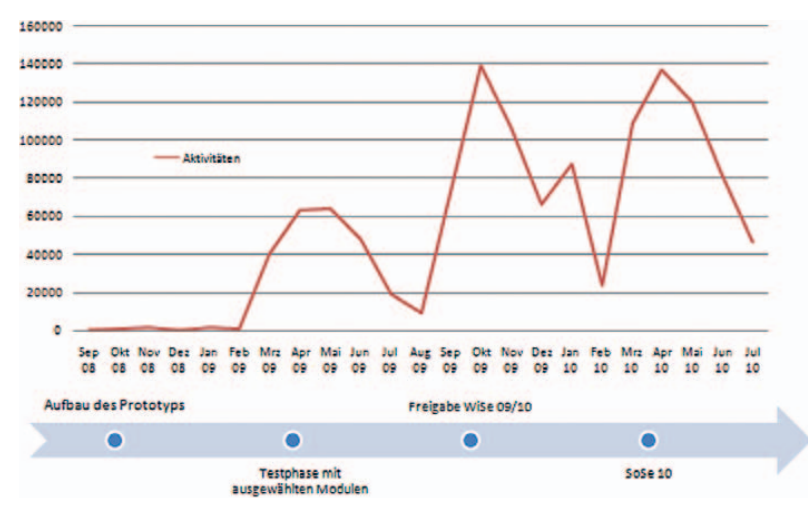

Abb. 2: Entwicklung der Zugriffe im Projektverlauf

Der Projektverlauf zeigt sich in der Entwicklung der Zugriffe (Aktivitäten) in Abb. 2: So waren in der Aufbauphase des Prototypen noch recht wenige Zugriffe zu verzeichnen. In dieser Phase wurde nur eine einzige Lehrveranstaltung zu Testzwecken durchgeführt. Im Sommersemester 2009 wurde die Testphase auf weitere 10 Kurse mit ca. 600 Studierenden ausgeweitet und entsprechend höhere Zugriffszahlen erzielt. Seit dem Wintersemester 2009/2010 ist die E-Learning-Plattform für alle Lehrveranstaltungen freigegeben und in die Phase des Normalbetriebes übergegangen. Die höchsten $\mathrm{Zu}$ griffszahlen sind bislang zu Semesterbeginn und die niedrigsten Zugriffszahlen in der vorlesungsfreien Zeit zu verzeichnen.

Bis zum Ende des Sommersemesters 2010 wurden insgesamt 3.088 Nutzer und Nutzerinnen registriert. Wird die Anzahl der Registrierungen um inaktive oder nur geringfügig genutzte Accounts bereinigt, wurde die Plattform von rund 2.100 Personen regelmäßig genutzt, die dort zwischen Januar und Juli 2010 durchschnittlich 289 Aktivitäten pro Kopf auslösten. Die gezählten Aktivitäten verteilten sich auf rund 319 aktive Kurse [4], wobei jeder Kurs durchschnittlich 2.722 Zugriffe (Aktivitäten) zu verzeichnen hatte. Damit wurde bislang ca. die Hälfte der Hochschulangehörigen im Bereich Lehre an der TH-Wildau erreicht. Im aktuellen Wintersemester 2010/2011 zeigt sich mit den Neuimmatrikulationen eine weitere deutliche Steigerung des Nutzeranteils. Insgesamt sprechen die reinen Zugriffszahlen dafür, dass sich die noch recht junge Plattform inzwischen an der TH Wildau etabliert hat und auch relativ intensiv genutzt wird.

Hinweise auf die Qualität der Nutzung finden sich in der Verteilung der Zugriffe auf die einzelnen Module (Aktivitäten), die sich grob zu den bereits genannten Kernfunktionen des E-Learnings zuordnen lassen. Seit Übergang in den Normalbetrieb im September 2009, ei-

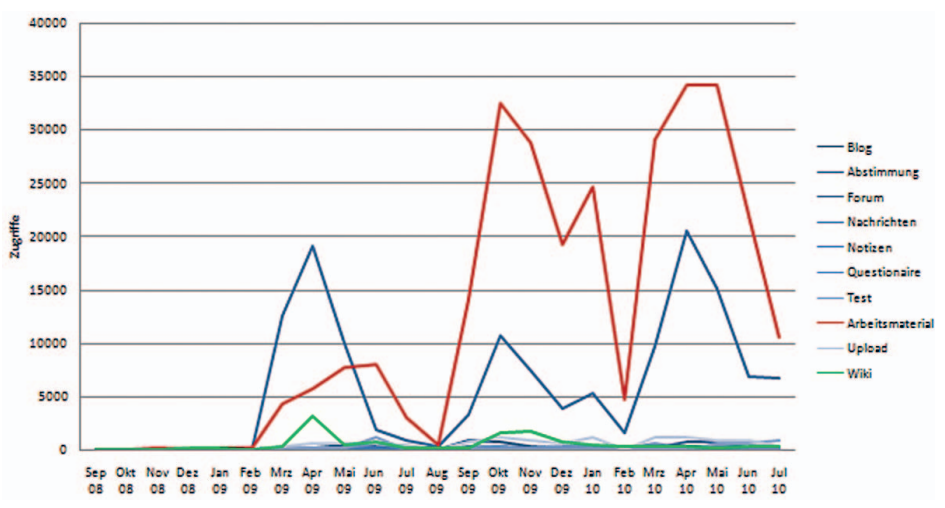

Abb. 3: Verteilung der Zugriffe nach Modul/Aktivitäten

ner wahrlich sehr kurzen Implementierungszeit, finden sich die höchsten Zugriffszahlen im Ressourcenmodul, d. h., der Bereitstellung und Nutzung von Arbeitsmaterialien (rote Linie in Abb. 3).

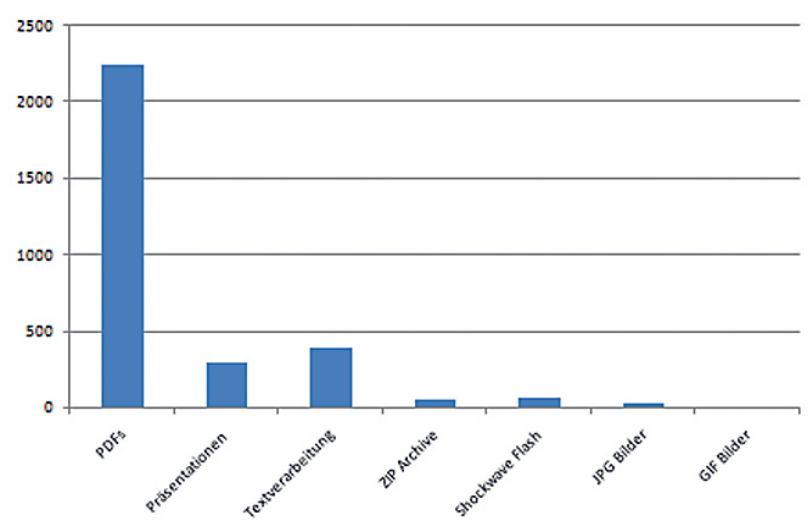

Abb. 4: Arbeitsmaterialien der Lernplattform

Bis Ende des Sommersemester 2010 sind insgesamt 4.037 Arbeitsmaterialien und Verlinkungen auf externe Quellen bereitgestellt worden. Von den 3.077 Arbeitsmaterialien, die in Form von Dateien auf dem Server abgelegt wurden, stellen PDF-Dateien, Präsentationen und Dokumente der Textverarbeitung den Löwenanteil (vgl. Abb. 4). Die starke Nutzung des Ressoucenmoduls und das Übergewicht von digitalen Dokumenten aus der »Offline Welt« deuten auf Learning by Distributing als dominierende Nutzungsform der Plattform. Diese Nutzungsform hat durchaus Vorteile, da Learning by Distributing die Präsenzlehre durch die komfortable Bereitstellung von Lehrmaterialien entscheidend stützt, bedeutet aber trotzdem , dass die Potenziale der Interaktivität und des kooperativen Arbeitens noch nicht voll genutzt werden.

Die Nutzung des Ressoucenmoduls gibt ebenso Hinweise auf Learning by Interacting, da über das Ressourcenmodul auf insgesamt 67 Flash-Dateien mit interaktiver Funktionalität verlinkt wird. Die Plattform bietet 
ferner interaktive Kurse, die über SCORM eingebunden sind und mit insgesamt 6.783 Zugriffen durchaus aktiv genutzt werden. Interaktion zeigt sich ebenfalls in der Nutzung der Upload-Funktion (12.162 Zugriffe), welche die Abgabe von Arbeitsleistungen der Studierenden erleichtert oder das Quiz-Modul (3.925 Zugriffe), das die Erstellung von Multiple Choice Tests ermöglicht.

Neben der dargestellten Nutzung des Ressourcenmoduls ist insbesondere die starke Nutzung der Diskussionsforen auffällig, was darauf hinweist, dass auch Learning by Collaboration auf der E-LearningPlattform eine Rolle spielt (blaue Linie in Abb. 3). Die Nutzung kollaborativer Tools wie Blogs (4.717 Zugriffe) oder Wikis (11.571 Zugriffe) sind in dieser frühen Nutzungsphase nicht unerheblich, sodass davon auszugehen ist, dass die Potenziale der Kollaboration und Kommunikation über die E-Learning-Plattform bereits jetzt in einigen Lehrveranstaltungen Berücksichtigung erfährt.

Aus der Datenanalyse der Lernplattformnutzung an der TH Wildau sind deutliche Ansätze einer neuen Vielfalt an Lehr- und Lernformen zu erkennen. Um diese Ansätze weiter zu entfalten und damit eine neue Qualität der Lehre zu sichern, ist aktuell und zukünftig die systematische Einbindung von Blended Learning in unterschiedliche Lernszenarien zu unterstützen und zu entwickeln (vgl. Abb. 5).

\section{Von der Präsenzlehre zum Blended Learning}

\subsection{Blended Learning als Veränderung der Lehrkultur und Lernformen}

In den Projekten Virtual Campus und learn@wildau wurde mit der erfolgreichen Einführung einer E-Learning Plattform die technische Infrastruktur als Voraussetzung für die Umsetzung innovativer Lehre geschaffen. Die aktuelle Nutzung der E-Learning Plattform zeigt zwar, dass die interaktiven und kollaborativen Potenziale der Plattform schon in Ansätzen genutzt werden, aber von der Umsetzung neuer Lernformen in einem »Blended Learning Setting« noch nicht die Rede sein kann. Vor diesem Hintergrund wird die Erkenntnis bedeutsam, dass der konsequente Weg in das Blended Learning immer auch Innovationen in der Lehre einschließt und damit auch zu Veränderungen in der Lehrkultur und der Lernformen führen muss.

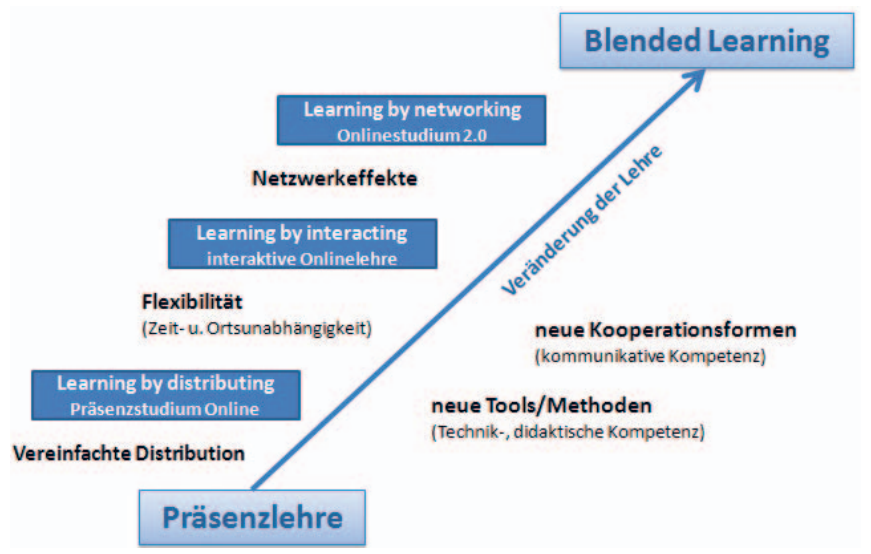

Abb 5 Die Stufen des Blended Learning

\subsection{Potenziale des Internets als Impulsgeber und Voraussetzung}

Ausgangspunkt sind die Potenziale des Internets, die vermittelt durch eine Lernplattform zum Impulsgeber und zur Voraussetzung für neue Lernformen werden können. Die Hauptfunktionen des E-Learning lassen sich als hierarchische Stufen des Blended Learnings darstellen, wobei mit jeder Stufe besondere Potenziale, aber auch besondere Anforderungen an Lehrende und Studierende verbunden sind (vgl. Abb. 5).

Learning by Distributing stellt in dieser Hierarchie die einfachste Variante des E-Learning dar, die auch die traditionellen Lernformen unberührt lässt, solange die Bereitstellung der Materialien in klassischer Rollenverteilung durch die Lehrenden und die Nutzung (bzw. der Download) durch die Studierenden erfolgt (Präsenzstudium Online). Die Möglichkeit der kostengünstigen und einfachen Verteilung von Lernmaterialien kann jedoch ebenso andere Lernparadigmen unterstützen, wenn Learning by Distributing für Gruppen- und Projektarbeit genutzt wird. Bereits auf dieser Stufe werden besondere - nicht als selbstverständlich vorauszusetzende - Anforderungen an das Qualifikationsprofil der Lehrenden und Studierenden gestellt. So müssen beide Zielgruppen mit den wichtigsten Funktionen der E-Learning Plattform vertraut sein. Die Bereitstellung von Lehrmaterialien stellt zudem die Lehrenden vor das Problem, Digitale- oder Printmedien der Offlinewelt für die Onlinewelt aufzubereiten und auf der Plattform in eine sinnvolle Kursstruktur zu bringen. Mit dem aktuellen Supportangebot der Lernplattform kann dieser Level der didaktischen Infrastruktur durch Video-Tutorials, Kurzschulungen sowie Telefon oder EMail Support weitestgehend abgedeckt werden. 
Mit dem Level des Learning by Interacting werden bereits die interaktiven Potenziale eines OnlineAngebotes genutzt. Der Gewinn liegt in der größeren Zeit- und Ortsunabhängigkeit, was insbesondere bei berufsbegleitendenden Bildungsangeboten aber auch für ein familienfreundliches Studieren Vorteile bietet. Zeit- und Ortsunabhängigkeit bedeutet allerdings, dass die Lehrenden und Lernenden mit einer veränderten Kursorganisation und der Nutzung interaktiver Tools konfrontiert werden, die nicht nur beherrscht, sondern auch in einen didaktischen Rahmen eingebettet werden müssen.

Mit dem Level des Learning by Collaboration erschließen sich die Netzwerkeffekte des Online-Lernens. Dieser Level ist nicht nur mit besonderen Tools, sondern auch mit besondere Lernformen und Methoden verbunden, die einen Bruch mit der an Hochschulen tradierten Lehre darstellen. Learning by Collaboration knüpft in der Regel an neuere didaktische Konzepte an, die nicht auf Frontalunterricht, sondern auf aktivierende Lernformen setzen. In der Hochschullehre würde das bedeuten, dass beispielsweise die Vorlesung als klassische Lernform zugunsten projektorientierter Konzepte wie Work-Based Learning oder ProblemBased Leaning zurückgedrängt wird. Inspiration und Anregung wird zudem im kollaborativen Arbeiten des "Mitmach-Web« gesucht [5], das als innovativer Weg in das lebenslange Lernen bereits diskutiert wird und seine Anhänger findet. Es gilt dabei, Lernende aus der Rolle des passiven Konsumenten in die Rolle des aktiv Gestaltenden zu heben und diesen Lernprozess kritisch zu begleiten. Unter dem Begriff »Social Media « zusammengefasst, sollen die aktiv Gestaltenden in »Communities« die Lernprozesse zu mehr Transparenz, Offenheit, Partizipation und Kollaboration führen. (Scholl u. a. 2010) In der Freizeit beteiligen sich bereits viele Studierende an Social Media Plattformen wie StudiVZ oder Facebook und lernen darüber intuitiv und informell. In Unternehmen ist Social Media Kommunikation - trotz mangelhafter Strategieentwicklung und oft fehlender Guidelines - aktuell und auch zukünftig ein bedeutendes Thema (Fink et al. 2010), und selbst moderne Verwaltungen kommen mit Government 2.0/ Open Government daran nicht vorbei (Appel 2010). Daher ist anzunehmen, dass die zukünftigen Arbeitgeber unserer Absolventen neben Fachkompetenz und Soft Skills auch Fähigkeiten und Fertigkeiten im Bereich von Social Media erwarten.

\subsection{Blended Learning als Transformation}

der Hochschullehre

Eine BL-Strategie beinhaltet neben der Didaktik auch die Organisationsstrukturen und Abläufe, die den Rahmen und Weg in eine neue Qualität der Lehre beschreiben. In der Hochschullehre und daran anknüpfender Angebote geht es in der Regel um die Transformation der Einzelmodule eines Studienganges vom OfflineModul zum Blended-Learning-Modul.

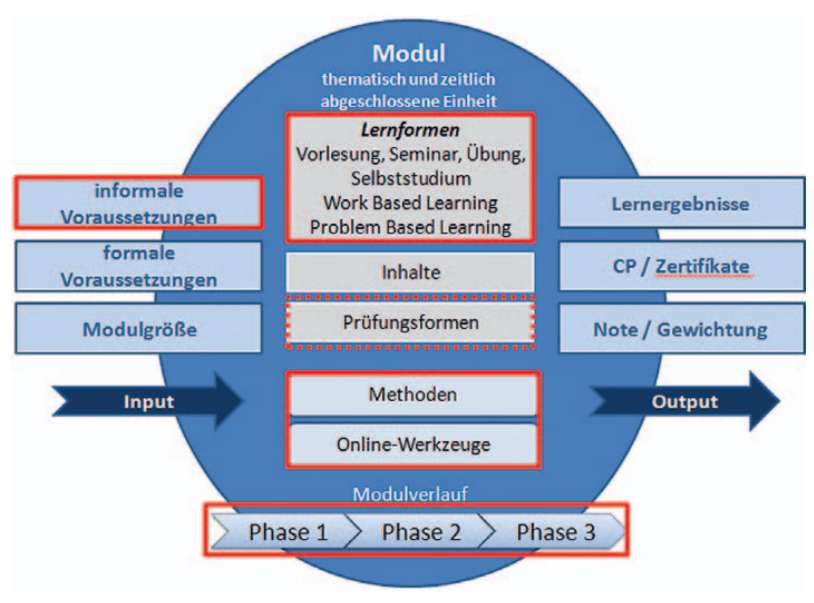

Abb. 6: Ansätze der Transformation im Studienmodul, Quelle: (Achterberg u. a. 2010)

Der Transformationsprozess berührt nicht alle Elemente eines Moduls: Konstant bleiben in der Regel die formalen Festlegungen eines Moduls, wie die fachlichen Voraussetzungen, die Gewichtung der Note oder die Anzahl der Creditpoints. Unberührt bleiben auch die Inhalte und die Lernergebnisse. Gegenstand des Transformationsprozesses ist und zur Disposition steht hingegen der Modulverlauf, die Lernformen, Methoden und Werkzeuge. Dies berührt zusätzlich das Qualifikationsprofil der Lehrenden und Studierenden. (rot umrandete Bereiche der Grafik, Abb. 6):

- Modulverlauf: Die Umsetzung von Blended Learning bietet die Chance, die orts- und zeitgebundenen Teile der Präsenzveranstaltung durch Online-Angebote zu ersetzen. Die auf das Vollzeitstudium mit wöchentlichen Präsenz-Veranstaltungen und fester Wochenstundenzahl ausgerichteten Studienmodule können auf diese Weise flexibilisiert werden, sodass über diese Module auch berufsbegleitende Angebote für neue Zielgruppen erschlossen werden können. Flexibilisierung ist jedoch in der Regel mit einer Veränderung des Modulverlaufs verbunden. In der Gestaltung des Modulverlaufs müssen die Abfolge und Verteilung von Online- und Präsenzzeiten, sowie die Betreu- 
ungsmodalitäten neu festgelegt werden. Ein großes Potenzial der Online-Lehre besteht somit in flexibleren Angeboten mit veränderten Präsenzzeiten.

- Lernformen: Mit der Ergänzung oder Ersetzung von Präsenzzeiten stehen gleichsam die klassischen, auf die reine Präsenzlehre zugeschnittenen Lernformen wie Vorlesung, Seminar oder Übung zur Disposition. Weil die angestrebten Lernergebnisse eines Moduls unabhängig von der gewählten Lernform sind, bleiben die Lernziele eines Studienmoduls davon unbetroffen. Um jedoch die Potenziale des Online-Lernens im Sinne einer Nachhaltigkeit für alle Lerntypen auszuschöpfen, sollten die Lernformen im Sinn von BL modifiziert werden.

- Methoden und Werkzeuge: Mit der Ergänzung und Ersetzung von Teilen der Präsenzlehre durch Formen der Online-Lehre ist die Nutzung von Online-Werkzeugen und spezieller Methoden verbunden, die eine optimale Verbindung von Präsenz- und Onlinelehre erst möglich machen. Gefordert ist, technische Kompetenz und zusätzliches Methodenwissen in der Anwendung der Online-Werkzeuge integrativ einzubinden.

- Qualifikationsprofil: Die Nutzung von Online-Medien zwingt die Lehrenden dazu, das auf die klassische Präsenzlehre abgestimmte Methodenrepertoire um methodische Elemente zu erweitern, die auf die Online-Lehre abgestimmt sind. In Abhängigkeit von den verwendeten Methoden und Online-Tools erfordert die konsequente Nutzung der Online-Medien ebenfalls zusätzliche kommunikative und technische Kompetenzen. Die Erweiterung des Qualifikationsprofils betrifft nicht nur die Lehrenden, sondern auch die Studierenden/Lernenden, da der Einsatz von Online-Medien und selbstorganisierter Lernformen erhebliche Anforderungen stellt.

In der Konsequenz fordert die erfolgreiche Umsetzung eines BL-Konzeptes, eine umfangreiche technische und didaktische Unterstützung inklusive benutzerfreundlicher Schnittstellen, der Bereitstellung eines Methodenpools, Erfahrungsberichten und vielfältigen Möglichkeiten der Diskussion und des Austausches.

\subsection{Herausforderungen beim Aufbau einer didaktischen Infrastruktur}

Der Aufbau einer didaktischen Infrastruktur soll helfen, die Potenziale und Kommunikationswerkzeuge des Internets über neue Lernformen und Methoden in eine neue Qualität der Lehre zu führen. Die Qualität der Lehre ändert sich dabei in den Lernformen, Methoden, Kooperationsstrukturen und der Kursorganisation unter Beibehaltung der ursprünglichen Lernziele. Anders formuliert, die Studierenden erwerben unter einer BL-Strategie dasselbe Fachwissen und dieselben Fachfähigkeiten auf gleichem Niveau, jedoch mit zusätzlichen Lerneffekten und Fertigkeiten sowie mit allen Vorteilen der orts- und zeitunabhängigen Online-Lehre. Eine didaktische Infrastruktur, muss für den Transformationsprozess von der klassischen Präsenzlehre zum Blended Learning die Voraussetzungen schaffen und die dazu notwendigen Mittel bereitstellen. In Anlehnung an die Stufen und Funktionen des E-Learnings und den Ansatzpunkten der Transformation in der Hochschullehre stellt sich die Anforderungen und Elemente einer didaktischen Infrastruktur wie folgt dar (vgl. Abb. 5):

- Mit jeder Stufe der Hauptfunktionen des E-Learnings wächst das Potenzial des Online-Lernens von der vereinfachten Distribution von Lernmaterialien bis hin zur Flexibilisierung durch die Zeit und Ortsunabhängigkeit interaktiver Angebote oder der Nutzung von Netzwerkeffekten im kollaborativen Lernen.

- Mit jeder Stufe steigen gleichzeitig der Änderungsbedarf im Modulverlauf, in der Auswahl der Lernformen sowie der Bedarf an neuen Methoden und Werkzeugen.

- Mit jeder Stufe steigen infolgedessen die Anforderungen an das Qualifikationsprofil der Lehrenden und Lernenden sowie an die Selbst- und Gruppenorganisation.

Bei der Einführung von Blended Learning handelt es sich um einen kontinuierlichen Entwicklungsprozess, dessen Pilotprojekte und Best Practice Beispiele in einer Strategie für den notwendigen Aufbau einer didaktischen Infrastruktur mündet. Die organisatorische Etablierung von Blended Learning sollte gemeinsames Ziel sein auch wenn der Aufbau einer didaktischen Infrastruktur zusätzliche Ressourcen und Anstrengungen erfordert, die durch den technischen Support allein nicht geleistet werden können.

Im Zuge dieses Wandels der Hochschullehre wird es auch zu einer Rollenveränderung der Lehrenden und Lernenden kommen. Für die Lehrenden wird dies - zumindest anfangs - mit Mehrarbeit verbunden sein, sei es für die Entwicklung neuer didaktischer Konzepte oder sei es für die Erstellung onlinegerechter Lernmaterialien. Das kann nur gelingen, wenn die Lehrenden durch eine organisatorisch eingebettete didaktische In- 
frastruktur praktische Hilfe und Unterstützung bekommen. Voraussetzung dafür ist deren flächendeckende institutionelle Einbindung an der Hochschule, die Raum für die begleitende Entwicklung weiterführender Strategien schafft. Dabei sollte die BL-Strategie bis auf die Frage der Anrechenbarkeit für Lehrende operationalisiert und beantwortet werden.

\section{Fazit und Ausblick}

Wie radikal muss Blended Learning umgesetzt werden, um in der Hochschullandschaft und am Markt bestehen zu bleiben? Welche Anwendungsbereiche eigenen sich besonders dazu, Blended Learning Settings zu entwickeln? Wie sehen sinnvolle Konzepte der einzelnen BL-Ausprägungen (z. B. problembasiert, projektbasiert oder aktionsbasiert) konkret für die einzelnen Studiengänge der TH Wildau aus? Welche Fähigkeiten und Fertigkeiten müssen Lehrende und Lernende entwickeln, um in ihren neuen Rollen bestehen zu können? Diese Fragen und die Beschreibung des Prozesses von der Präsenzlehre zum Blended Learning zeigen, dass ein solches Vorhaben ernsthafter Anstrengungen aller Seiten bedarf und eine deutliche Veränderung der Lernkultur notwendig macht, die Überzeugungsarbeit erfordert. Zusätzlich bedarf es verstärkter Kleingruppenarbeit und anderer Raumnutzungen, die organisiert werden müssen, und sicherlich erweiterter personeller Ressourcen zur technischen und didaktischen Unterstützung. Insofern verwundert es nicht, dass erfolgreiche BL-Konzepte mit einer organisatorischen Strategieentwicklung einhergehen müssen, wenn man die Umsetzung einer neuen Qualität der Lehre ernst nimmt.

Im Juni 2010 wurde zur Unterstützung das Wildau Institut für innovative Lehre, lebenslanges Lernen und gestaltende Evaluation (WILLE) unter dem Dach des TWZ e.V. gegründet. Zweck des Instituts ist die Förderung des lebenslangen Lernens und die Verbesserung der Lehr- und Lernqualität unter Einsatz innovativer Lehrmethoden und Technologien. Ziel ist die Umsetzung und Institutionalisierung von Blended Learning in der Hochschullehre und akademischen Weiterbildung sowie in ausgewählten Anwendungsfeldern der beruflichen Fortbildung. Das Institut fördert und betreibt zu diesem Zweck die wissenschaftlich fundierte Forschung, Entwicklung und Evaluation innovativer Lernkonzepte, die eine didaktisch sinnvolle Verknüpfung von traditioneller Präsenzlehre und modernen
Formen des Online-Lernens (Blended Learning) anstreben. Das Institut nimmt darüber hinaus Informations-, Weiterbildungs- und Beratungsaufgaben für den akademischen und berufspraktischen Nachwuchs in und außerhalb der Region wahr. Das Institut arbeitet mit Hochschulen, Ministerien, Behörden, Unternehmen, Verbänden, Kommunen, Weiterbildungsträger und anderen Institutionen, insbesondere der Technischen Hochschule Wildau [FH] und ihren Kooperationspartner, eng zusammen. Zielgruppen sind $\mathrm{u}$. a. Hochschullehrende, freie Dozenten, Weiterbildungseinrichtungen und berufstätig Lernende aus allen Bereichen. Das Institut verfolgt gemeinnützige Ziele und Zwecke und strebt deshalb nur die Deckung der entstandenen Kosten an.

An der TH Wildau selbst wird in dem Zielvereinbarungsprojekt "Service Point Qualifizierung- Blended Learning als Schlüssel zwischen Beruf und Aus- bzw. Weiterbildung am Beispiel der Logistik « mit einer wissenschaftlichen Mitarbeiterstelle den Entwicklungsaspekten eines »Blended Learning Settings« nachgegangen. Hier wird versucht, die notwendige Strategie zur hochschulweiten Einführung von Blended Learning mit konkreten Konzepten aufzuzeigen und beispielhaft in der Logistik umzusetzen. Unser Beitrag verdeutlicht die dahinterliegende Komplexität und die knappen personelle Ressourcen. Auch sind keineswegs alle Fragen hinsichtlich der Akzeptanz und Anrechnung geklärt. Die TH Wildau kommt trotzdem voran, vor allem wegen der professionellen Kompetenz der Projektmitarbeiter und der Synergieeffekte durch weitere Drittmittelprojekte.

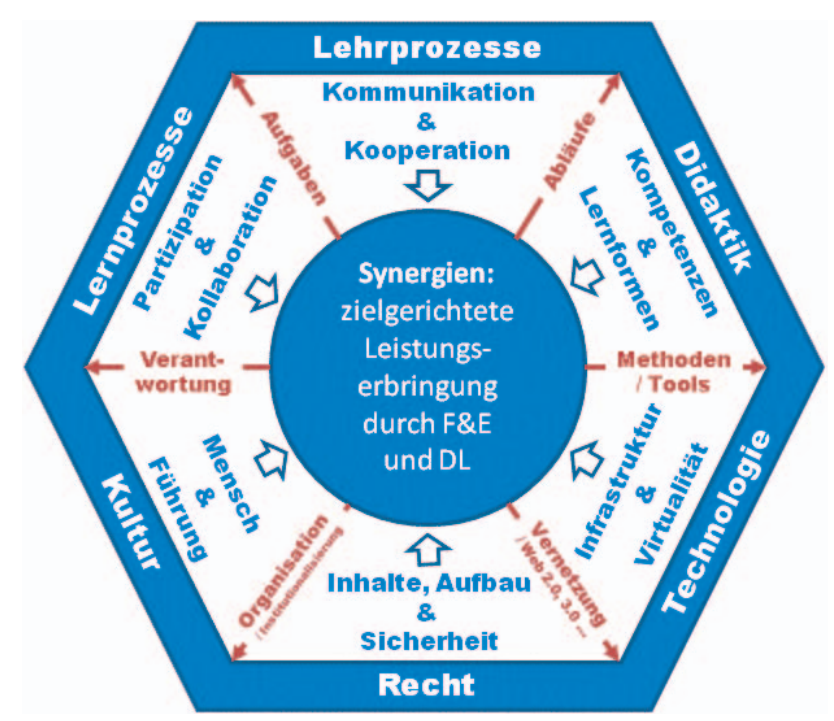

Abb. 7: Gesamtkonzept zur neuen Qualität in der Lehre 
Die Abb. 7 fasst das bisher von den Autorinnen entwickelte Gesamtkonzept in seinen vielfältigen Aspekten und synergetischen Potenzialen zusammen: Neben den bereits im Artikel angesprochenen Lehr- und Lernprozessen, der Didaktik und der Technologie fließen in dieser Darstellung auch Aspekte der (Lern-/Lehr-) Kultur und des Rechts ein, die gesondert zu behandeln sind. Mit den dargestellten Wechselwirkungen versucht die Abbildung den Ausblick auf die zukünftigen Anforderungen grafisch zu fassen. Im Mittelpunkt der Abb. 7 stehen die Synergieeffekte durch Forschung und Entwicklung (F\&E) in (Drittmittel-) Projekten und die Erfahrungen aus den angebotenen Dienstleistungen (sowohl innerhalb der Direkt- und Fernstudiengänge als auch z. B. über das Institut WILLE). In diese Aktivitäten fließen alle bisherigen Überlegungen zu einer BLStrategie ein (blau-weiße Pfeile in Abb. 7) und werden in den Forschungsprojekten untersucht, für Aus-, Fortund Weiterbildung weiterentwickelt und in Piloten getestet sowie konkret gestaltet.

Eine BL-Strategie der höchsten Stufe (vgl. Abb. 5) umfasst nach Abb. 7 (im äußeren Rand angeordnet) integrativ die Lehr- und Lernprozesse mit Kommunikation und Kooperation der Lehrenden sowie Partizipation und Kollaboration der Lernenden. Zunehmend wird dazu die Lernkultur einer Organisation zu hinterfragen sein, die den lernenden Menschen in seiner Wissensentwicklung unterstützen soll und neue Anforderungen an die Führungskräfte stellen wird. Die technologische Infrastruktur als Basis neuer Virtualität muss flankiert werden durch einerseits die Einhaltung und Ausgestaltung rechtlicher Aspekte einer umfassenden Informationssicherheit (wie IT-Sicherheitsmaßnahmen, Datenschutz und Datensicherheit, Urheberrecht, Regelungen etc.) und andererseits durch eine bereits beschriebene didaktische Infrastruktur, die die Entwicklung der didaktischen Kompetenzen der Lehrenden und den Einsatz der vielfältigen Lernformen unterstützen hilft, indem aus der Forschung \& Entwicklung heraus Beispiele passender Abläufe, mit nutzbaren Methoden und Tools für die Praxis zur Verfügung gestellt werden. Damit werden die im Artikel besprochenen »Blended Learning Settings« konkret machbar, die Verantwortlichkeiten der Akteure zur Aufgabenbewältigung verdeutlicht und die Abläufe modernisiert.
Diese perspektivische Anforderungsdarstellung (Abb. 7) im Blick sollten an der TH Wildau die nächsten konkreten Umsetzungsschritte verstärkt der institutionellen Stärkung einer didaktischen Infrastruktur gewidmet werden.

\section{Anmerkungen}

[1] Aufstieg durch Bildung - die Qualifizierungsinitiative der Bundesregierung: Bildungsgipfel am 22. Oktober 2008 in Dresden. Auf dem Bildungsgipfel haben Bund und Länder Aktivitäten zur Förderung und Unterstützung von Bildung über den gesamten Lebensweg von der frühkindlichen Bildung bis zur Weiterbildung im Beruf vereinbart.

[2] Das COMBLE Projekt wurde bereits im Rahmen der Wissenschaftlichen Beiträge vorgestellt. (Niemczik et al. 2008) http://www. comble-projec t.eu, 15.09.2010.

[3] Die Aktivitäten der Administratoren/innen sind in dieser Zahl bereits herausgerechnet.

[4] Auch diese Zahl ist um Kurse mit niedrigen Zugriffszahlen bereinigt, gezählt wurden nur Kurse mit mehr als 30 Zugriffen.

[5] Berger, A. (2007), »Mitmach-Web als Millionärsgarantie«im Stern, http://www.stern.de/digital/online/verkauf-von-facebookanteilen-mitmach-web-als-millionaersgarantie-601046.html, 12.09.2010

\section{Literatur}

Achterberg, B., Büschenfeldt, M. und Vossel, M. (2010): Modularisierung, HDL Fachtagung.

Appel, J. (2010): »Web 2.0 - und die Chancen für die öffentliche Verwaltung in Blogs, Podcast, Social Networks und Co.«, Entwicklung eines Leitfadens, Diplomarbeit, FB WVR, Studiengang Verwaltung und Recht, TH Wildau.

BMBF (2008): Lebenslanges Lernen, http://web.archive.org/ web/20080822031749/http://www.bmbf.de/de/411.php, Zugriff 02.09.2010

Fink, S., Zerfaß, A. (2010): Social Media Governance 2010, Universität Leipzig / Fink \& Fuchs PR, http://www.socialmediagovernance. eu, Zugriff 15.8.2010.

Hof, C. (2009): Lebenslanges Lernen: Eine Einführung, Kohlhammer, Stuttgart.

Kepser, M. (2010): E-Learning an der Hochschule. In: Neue Impulse in der Hochschuldidaktik, Springer, http://beat.doebe.li/bibliothek/ t12006.html, Zugriff 07.09.2010.

Niemczik, C., Brenstein, E. und Scholl, M. (2008): COMBLE: Community of Integrative Blended Learning Experts", Wissenschaftliche Beiträge 2008, 87-93.

Sauter, A., Sauter, W., Bender, H. (2004): Blended learning: effiziente Integration von E-Learning und Präsenztraining, 2., überarb. Auflage, Luchterhand, Unterschleissheim/München.

Scholl, M., Brenstein, E. und Niemczik, C. (2008): Ready for Blended Learning? - Ein ganzheitlicher Ansatz. In: 5. Beckmannkolloquium, Wismar, 2008, http://www.comble-project.eu/Plone/whatmore/DISS-Conference-Paper-Ready-for-BL.pdf/at_download/file, Zugriff 06.09.2010 
Scholl, M., Niemczik, C. und Büschenfeldt, M. (2010): Learning Communities im Web 2.0 am Beispiel von Methopedia - Inspirationen für die Lehre?, Wissenschaftliche Beiträge 2009/2010, 23-32.

Schulmeister, R. (2005): Kriterien didaktischer Qualität im ELearning zur Sicherung der Akzeptanz und Nachhaltigkeit. In: Euler, D.: E-Learning in Hochschulen und Bildungszentren., Oldenbourg, München, http://www.google.de/firefox?client=firefoxa\&rls=org.mozilla:de:official, Zugriff 07.09.2010

\section{Autoren}

Prof. Dr. Margit Scholl

Wirtschafts- und Verwaltungsinformatik

FB Wirtschaft, Verwaltung und Recht

Technische Hochschule Wildau [FH]

margit.scholl@th-wildau.de,www.th-wildau.de/scholl

\section{Dipl.-Soz. Maika Büschenfeldt}

Projektmitarbeiterin Projekt learn@wildau

(Zentrale Lernplattform Moodle)

FB Wirtschaft, Verwaltung und Recht

Technische Hochschule Wildau [FH]

maika.bueschenfeldt@th-wildau.de 